\title{
Effects of docosahexaenoic acid on learning and memory impairment induced by repeated propofol anesthesia in young rats
}

\author{
MING TIAN ${ }^{1,2^{*}}$, ZHI LI ${ }^{1,2^{*}}$, GAO WANG $^{2}$, WEIZHONG PAN ${ }^{2}$ and KEZHONG LI ${ }^{1,2}$ \\ ${ }^{1}$ Department of Anesthesiology, Shandong University School of Medicine, Jinan, Shandong 250012; \\ ${ }^{2}$ Department of Anesthesiology, Yantai Yuhuangding Hospital, Yantai, Shandong 264000, P.R. China
}

Received June 3, 2015; Accepted January 19, 2016

DOI: $10.3892 /$ etm.2016.3074

\begin{abstract}
The aim of the present study was to investigate the effects of docosahexaenoic acid (DHA) on the learning and memory ability of young rats exposed to propofol, and its underlying mechanisms. Sprague Dawley rats $(n=60)$ were randomly divided into six groups: Control group (group A); solvent control group (group B); propofol group (group C); low-dose DHA + propofol group (group D); medium dose DHA + propofol group (group E); and high-dose DHA + propofol group (group F). The Morris water maze (MWM) test was performed to evaluate the rats' learning and memory ability, and tissue samples from the hippocampi of the rats were obtained for biochemical analysis. The results of the MWM test revealed that DHA supplementation administered to young rats led to an evident decrease in the latency to find the maze platform, and a significant increase in the number of platform crossings in groups $\mathrm{E}$ and $\mathrm{F}$ compared with group $\mathrm{C}(\mathrm{P}<0.05)$. High-performance liquid chromatography indicated that glutamate concentration levels were significantly lower and $\gamma$-aminobutyric acid concentration levels were significantly higher in the hippocampi of group $\mathrm{E}$ and $\mathrm{F}$ rats treated with DHA compared with group $\mathrm{C}$ rats $(\mathrm{P}<0.05)$. Furthermore, DHA treatment alleviated the decrease in brain-derived neurotrophic factor levels $(\mathrm{P}<0.05)$, and superoxide dismutase $(\mathrm{P}<0.05)$ and glutathione peroxidase $(\mathrm{P}<0.05)$ activities induced by the administration of propofol. Additionally, DHA treatment decreased malondialdehyde levels in the hippocampi of rats $(\mathrm{P}<0.05)$. The aforementioned findings demonstrate that DHA was able to effectively improve learning and memory dysfunction induced by
\end{abstract}

Correspondence to: Dr Kezhong Li, Department of Anesthesiology, Shandong University School of Medicine, 44 Wenhua West Road, Jinan, Shandong 250012, P.R. China

E-mail: kezhongli0417@163.com

*Contributed equally

Key words: docosahexaenoic acid, propofol anesthesia, learning and memory ability, morris water maze, superoxide dismutase, glutamate repeated propofol-induced anesthesia in young rats. This data suggests that DHA may be a potential candidate for further preclinical studies aimed at treating postoperative cognitive dysfunction.

\section{Introduction}

Postoperative cognitive dysfunction (POCD) refers to the neurocognitive dysfunction caused by anesthesia, which is a common postoperative neurological complication. The clinical symptoms include patients undergoing personality changes days and even several weeks postoperatively, with decreased memory, attention, social adaptability, cognitive ability and comprehension ability. Long-term cognition disorders are also possible and severely affect the patient's quality of life (1). Propofol (2,6-diisopropylphenol) is an intravenous anesthetic widely used for the induction and maintenance of anesthesia in clinical practice. In recent years, studies concerning human memory dysfunction caused by propofol anesthesia have emerged (2-4). Propofol anesthesia (100 or $150 \mathrm{mg} / \mathrm{kg}$ ) was revealed to induce amnesia in rodents (5), in addition to decreased hippocampal cell proliferation and the induction of learning impairment in young rats (6). Propofol has been demonstrated to cause anesthesia, in particular in elderly and pediatric patients $(2,4,7)$. However, the mechanisms underlying memory impairment induced by propofol remain unclear. Thus, there is no effective treatment for postoperative cognitive dysfunction induced by propofol.

The hippocampus is located in the medial temporal lobe of the brain and is of considerable importance, as its primary function is associated with learning and memory. Damage to the structure of a neuron synapse may significantly affect the ability of learning and memory. Several studies have ascertained that exposure to propofol is associated with the deficit of cognitive and neurobehavioral functions by altering the release of neurotransmitters (8-11). Furthermore, substantial evidence in rats indicates that oxidative stress, including lipid peroxidation and protein oxidation, have an important role in causing damage to learning and memory ability induced by repeated propofol anesthesia. Alterations in antioxidant defense mechanisms also occur $(12,13)$. As oxidative stress is of importance to the propofol anesthesia process, antioxidant treatment may alleviate propofol-induced anesthesia-associated learning and memory impairments. 
Docosahexaenoic acid (DHA) is an essential n-3 polyunsaturated fatty acid that concentrates in membrane phospholipids at synapses and in retinal photoreceptors $(14,15)$. Clinical evidence indicates that term infants given formula supplemented with DHA plus arachidonic acid or DHA alone have improved cognitive ability (16). Animal studies have also revealed that a deficiency of DHA resulted in a poorer performance on cognitive and behavioral tests; conversely, supplementation with DHA led to the recovery of learning and memory-associated performance $(17,18)$. Furthermore, studies in animal models of neurodegenerative disorders indicate that the consumption of DHA produces beneficial effects by inhibiting oxidative stress and inflammation, and inducing the expression of cell survival genes (19-22).

The positive effects observed in brain tissues following the administration of DHA led the present study to hypothesize that DHA treatment may attenuate impairments in learning and memory abilities induced by repeated propofol-induced anesthesia in rats. To the best of our knowledge, the majority of studies have assessed the effects of DHA treatment on learning and memory ability in traumatic brain injury-induced subjects, whilst few have focused on the effects of DHA treatment on the impairment induced by repeated propofol anesthesia. Thus, the present study aims to evaluate the effects of DHA treatment on the impairment of learning and memory ability caused by repeated propofol-induced anesthesia in young rats based on Morris water maze (MWM) testing, and its association with amino acid neurotransmitters, antioxidant enzymes and brain-derived neurotrophic factor (BDNF).

\section{Materials and methods}

Chemicals. Propofol and Intralipid were obtained from Sigma-Aldrich China, Inc. (Shanghai, China). DHA was supplied by Rongcheng Baihe BioTechnology Co., Ltd. (Rongcheng, China). Superoxide dismutase (SOD; cat. no. A001-1) and glutathione peroxidase (GSH-Px; cat. no. A005) enzyme-linked immunoassay (ELISA) activity test kits were purchased from the Nanjing Jiancheng Bioengineering Institute (Nanjing, China). Malondialdehyde (MDA; cat. no. A003-1) and BDNF (cat. no. E0011) ELISA test kits were purchased from the USCN Life Science Inc. (Wuhan, China).

Animals. Sprague Dawley rats $(n=60 ; 40$ days old; half male and half female; weight, 150-200 g) were provided by the Experimental Animal Center of Suzhou Aiermaite Technology Co., Ltd. (Suzhou, China). Animals were kept in a controlled light room with a photoperiod of $12 \mathrm{~h}$ dark:light at a temperature of $22 \pm 2{ }^{\circ} \mathrm{C}$. All animals were allowed free access to standard laboratory chow and fresh water. All experimental procedures conducted in the present study were performed in accordance with the Guidelines for the Care and Use of Laboratory Animals of Yantai Yuhuangding Hospital. The study was approved by the ethics committee of Yantai Yuhuang Ding Hospital (Yantai, China).

Experimental protocol. Rats were randomly assigned to 4 groups ( $n=10$ per group) as follows: Group A, control group; group B, solvent control group; group C, propofol group; group D, low dose DHA + propofol group; group E, medium dose DHA + propofol group; and group F, high dose $\mathrm{DHA}+$ propofol group.

The animals in group A received $0.9 \% 10 \mathrm{ml} / \mathrm{kg}$ saline by intraperitoneal injection once daily for consecutive 5 days. The animals in group B received $10 \mathrm{mg} / \mathrm{kg}$ Intralipid by intraperitoneal injection once daily for consecutive 5 days. The rats in experimental groups C, D, E and F were administered $75 \mathrm{mg} / \mathrm{kg}$ propofol by intraperitoneal injection once daily for 5 consecutive days. Rats in experiment group D, E and $\mathrm{F}$ also received a single oral dose of $0.3,1$, and $3 \mathrm{~g} / \mathrm{kg}$ DHA, respectively, for 10 consecutive days prior to propofol exposure.

MWM test. Hippocampus-dependent spatial learning and memory were assessed via MWM testing, $12 \mathrm{~h}$ after the administration of propofol or Intralipid The rats were trained on five consecutive days with four consecutive trials occurring each day. The trials were conducted in a pool $(150 \mathrm{~cm}$ in diameter, $\left.25 \pm 2^{\circ} \mathrm{C}\right)$. Each rat was trained to find a hidden circular platform $(11 \mathrm{~cm}$ in diameter, $2 \mathrm{~cm}$ beneath the water in the southwestern quadrant). The rat was allowed a maximum of $120 \mathrm{sec}$ to reach the platform. If the rat was unable to find the platform within $120 \mathrm{sec}$, it was placed on the platform for $30 \mathrm{sec}$. Time taken to reach the platform was recorded. A probe trial was performed to assess memory $24 \mathrm{~h}$ after the last training session. During the probe trial, the hidden platform was removed from the tank and the rats were allowed to swim freely for $120 \mathrm{sec}$. Latency to find the maze platform (sec) and number of platform crossings were recorded.

Tissue sampling. Twenty-four hours after the MWM test was performed, the rats were sacrificed by an overdose of sodium pentobarbital $(30 \mathrm{mg} / \mathrm{kg})$. Then, the hippocampi of the rats were obtained and immediately divided into two sections of equal size. One section of tissue was used for the determination of amino acid neurotransmitters and the other section was stored at $-80^{\circ} \mathrm{C}$ for subsequent biochemical assays.

High-performance liquid chromatography (HPLC) analysis. The hippocampus was removed, weighed and placed in $1.5 \mathrm{ml}$ microcentrifuge tubes containing $1 \mathrm{ml}$ of chilled homogenisation buffer ( $0.1 \mathrm{M}$ citric acid, $0.1 \mathrm{M}$ sodium dihydrogen phosphate monohydrate, $5.6 \mathrm{mM}$ octane sulfonic acid, $10 \mu \mathrm{m}$ EDTA in $10 \%$ (v/v) methanol solution, pH 2.8 with $4 \mathrm{M} \mathrm{NaOH}$ ). Each sample was sonicated for $4 \mathrm{sec}$ (Sonoplus; Bandelin Electronic GbmH \& Co., Berlin, Germany), centrifuged at $18,620 \mathrm{x}$ g for $15 \mathrm{~min}$ at $4 \mathrm{C}$ and the supernatant stored at $-80^{\circ} \mathrm{C}$ until HPLC analysis. The concentration levels of glutamate (Glu) and $\gamma$-aminobutyric acid (GABA) were determined by HPLC with fluorescent detection, as described previously (23). An Agilent 1200 series HPLC system and Agilent ZORBAX Extend-C18 analytical column $(250 \times 4.6 \mathrm{~mm}, 5 \mu \mathrm{m}$; particle size; Agilent Technologies, Inc., Santa Clara, CA, USA) were used. The excitation and emission wavelengths were set to 355 and $450 \mathrm{~nm}$, respectively. Glu and GABA were quantified by comparison with the standard curves for each amino acid. The formula used for calculating Glu and GABA contents in the hippocampus is as follows: 

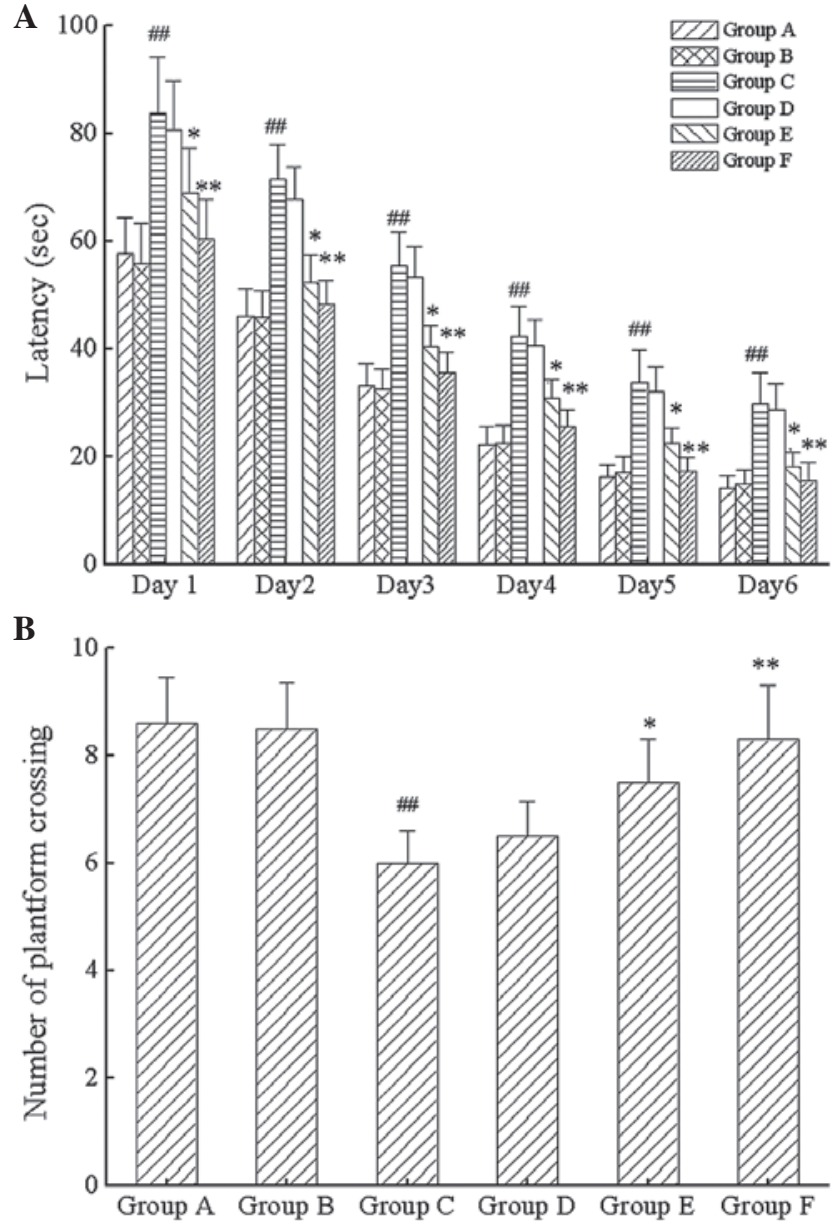

Figure 1. Effect of docosahexaenoic acid (DHA) treatment on (A) latency to find the maze platform and (B) the number of platform crossings. Group A, control group; group B, solvent control group; group C, propofol group; group $\mathrm{D}$, low dose $\mathrm{DHA}+$ propofol group; group $\mathrm{E}$, medium dose DHA + propofol group; and group F, high dose DHA + propofol group. Data are presented as mean \pm standard deviation $(n=10) .{ }^{\# \#} \mathrm{P}<0.01$ vs. group $\mathrm{A}$; ${ }^{*} \mathrm{P}<0.05,{ }^{* *} \mathrm{P}<0.01$ vs. group $\mathrm{C}$.

$$
\text { Glu or GABA }(\mu \mathrm{g} / \mathrm{g})=\frac{\operatorname{conc}_{\text {sample }}(\mu \mathrm{g} / \mu \mathrm{l}) \times \operatorname{vol}_{\text {sample }}(\mu \mathrm{l})}{\text { weight of hippocampus }(\mathrm{g})}
$$

where conc is concentration and vol is volume.

Biochemical analysis. Hippocampus tissues were homogenized using a glass homogenizer in a $100 \mathrm{mM}$ phosphate-buffered saline (PBS, pH 7.4; Beijing Biosynthesis Biotechnology Co., Ltd., Beijing, China) containing $1 \mathrm{mM}$ EDTA, phenylmethylsulfonyl fluoride (proteinase inhibitor; $1 \mathrm{mM}$ ) and phosphatase inhibitor cocktail (1:100 dilution) (all purchased from Beijing Biosynthesis Biotechnology Co., Ltd.). The mixture was centrifuged at $12,000 \mathrm{xg}$ for $30 \mathrm{~min}$ at $4^{\circ} \mathrm{C}$. The supernatant was collected and quantitatively assayed for the activities of SOD and GSH-Px, and the protein concentration of MDA and BDNF. The detection of these indicators was performed using ELISA kits, according to the manufacturer's protocol.

Statistical analysis. The data are expressed as mean \pm standard deviation. SPSS statistical software (version 16.0; SPSS,
A

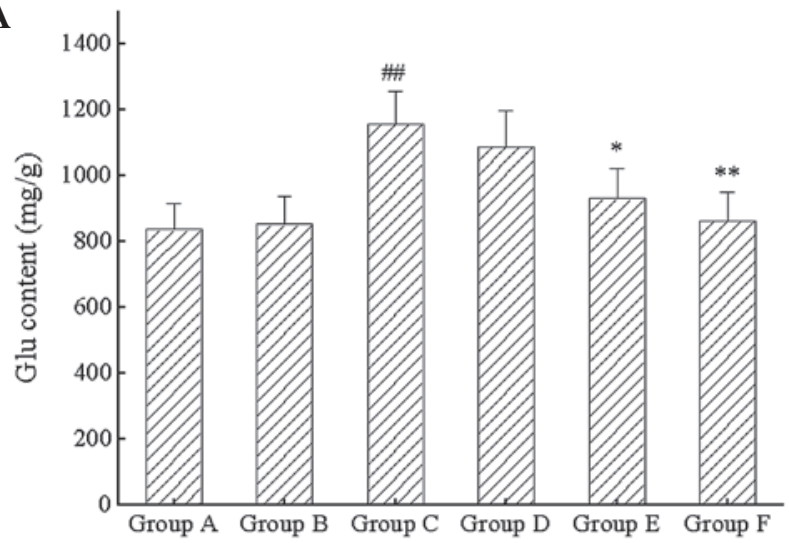

B

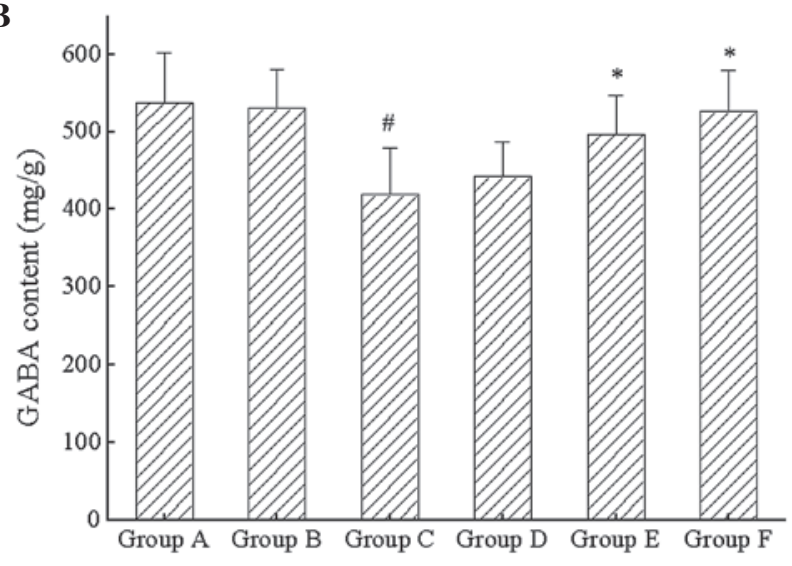

C

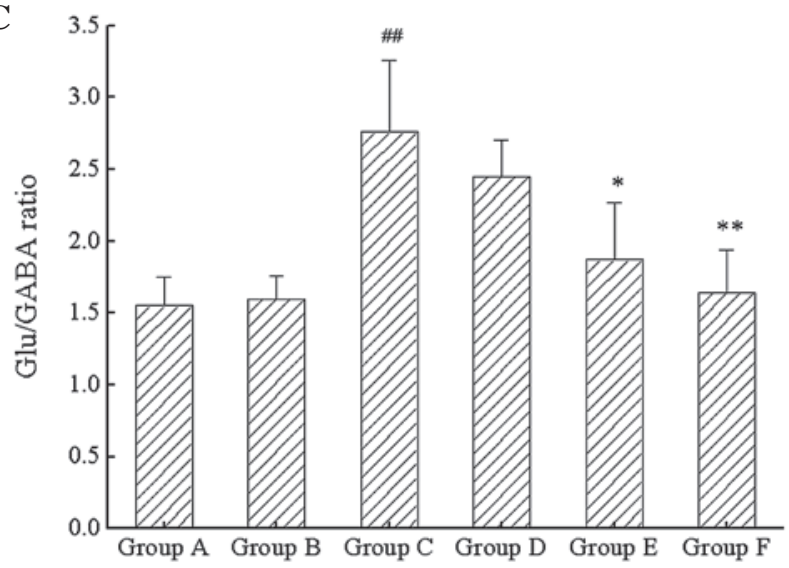

Figure 2. Effect of docosahexaenoic acid (DHA) treatment on the content of (A) Glu and (B) GABA, and (C) the ratio of Glu / GABA in the hippocampus. Group A, control group; group B, solvent control group; group C, propofol group; group D, low dose DHA + propofol group; group E, medium dose $\mathrm{DHA}+$ propofol group; and group F, high dose DHA + propofol group. Data are presented as mean \pm standard deviation $(\mathrm{n}=10)$. ${ }^{\#} \mathrm{P}<0.01$ vs. group $\mathrm{A}$; ${ }^{*} \mathrm{P}<0.05,{ }^{* *} \mathrm{P}<0.01$ vs. group C. Glu, glutamate; GABA, $\gamma$-aminobutyric acid.

Inc., Chicago, IL, USA) was employed for statistical analysis. Significant differences between the treatment and control groups were determined using one-way analysis of variance, followed by a Student-Newman-Keuls test. $\mathrm{P}<0.05$ was considered to indicate a statistically significant difference.

\section{Results}

Effects of DHA treatment on MWM testing. Fig. 1 revealed the effects of DHA treatment on latency to find the maze platform (Fig. 1A) and number of platform crossings (Fig. 1B) during 
A

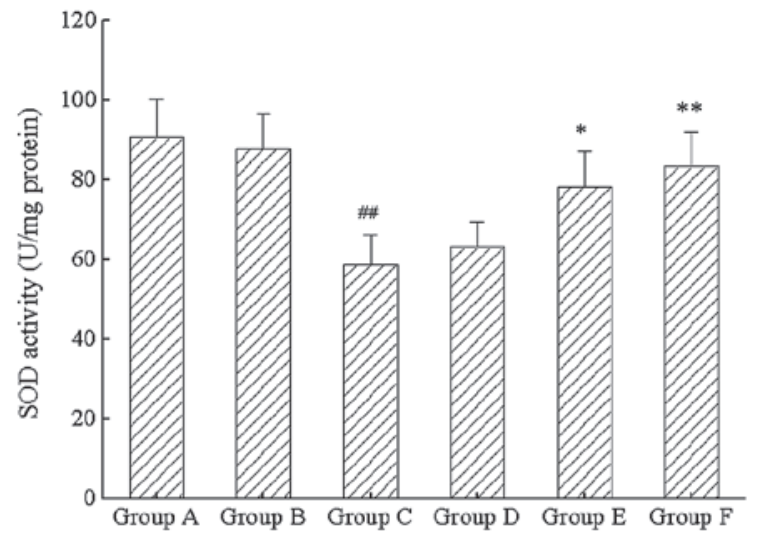

B

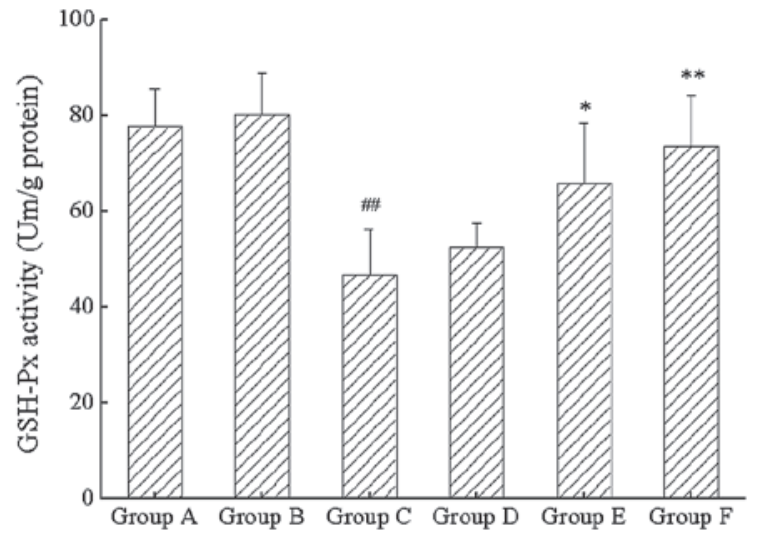

C

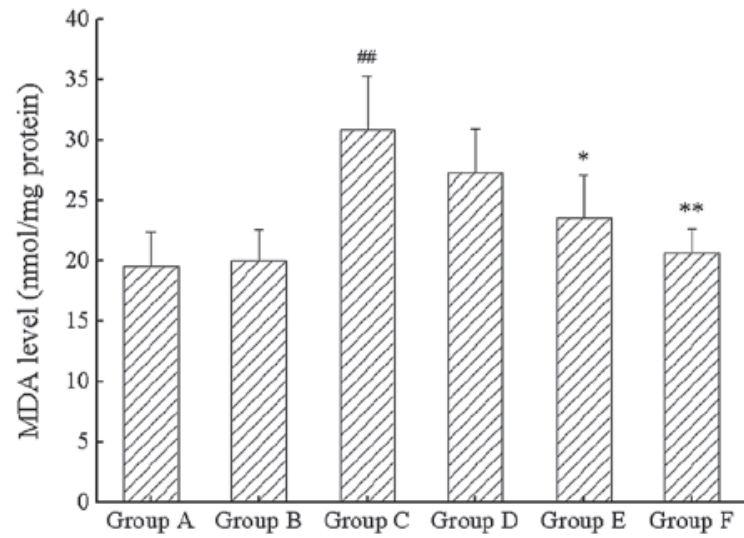

Figure 3. Effect of docosahexaenoic acid (DHA) treatment on the activity of (A) SOD and (B) GSH-Px, and (C) the protein concentration of MDA in the hippocampus. Group A, control group; group B, solvent control group; group $\mathrm{C}$, propofol group; group $\mathrm{D}$, low dose $\mathrm{DHA}+$ propofol group; group $\mathrm{E}$, medium dose DHA + propofol group; and group F, high dose DHA + propofol group. Data are presented as mean \pm standard deviation $(\mathrm{n}=10) .{ }^{\# \#} \mathrm{P}<0.01$ vs. group $\mathrm{A} ;{ }^{*} \mathrm{P}<0.05,{ }^{* *} \mathrm{P}<0.01$ vs. group $\mathrm{C}$. $\mathrm{SOD}$, superoxide dismutase; GSH-Px, glutathione peroxidase; MDA, malondialdehyde.

the MWM test. Administration of propofol in group C led to a significant elevation in latency to find the maze platform compared with group A $(\mathrm{P}<0.01)$. In comparison to group $\mathrm{C}$, groups $\mathrm{D}, \mathrm{E}$ and $\mathrm{F}$ exhibited reduced latency to find the maze platform, and the differences of group $\mathrm{E}(\mathrm{P}<0.05)$ and $\mathrm{F}$ $(\mathrm{P}<0.01)$ were statistically significant compared with group $\mathrm{C}$. Furthermore, with increasing dosage of DHA, the latency to find the maze platform of groups D, E and F were gradually reduced. This suggests that treatment with DHA may effectively and dose-dependently enhance the spatial learning and memory of young rats treated with propofol.

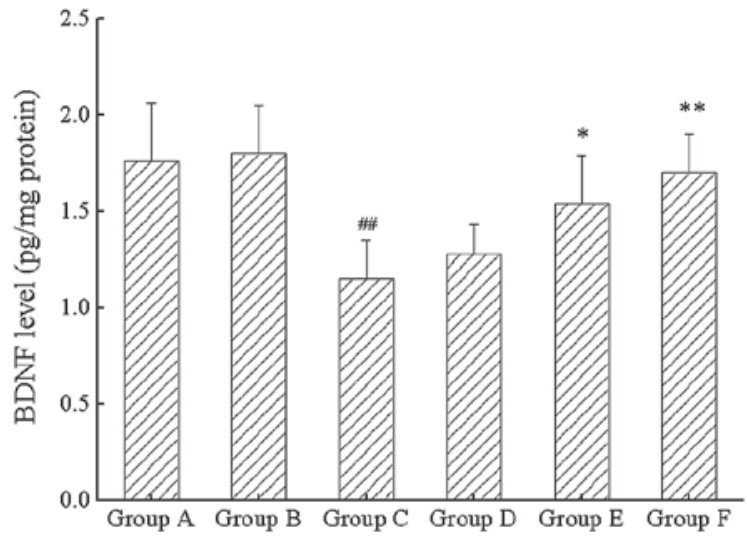

Figure 4. Effect of docosahexaenoic acid (DHA) treatment on the protein concentration level of BDNF in the hippocampus. Group A, control group; group B, solvent control group; group C, propofol group; group D, low dose $\mathrm{DHA}+$ propofol group; group E, medium dose DHA + propofol group; and group F, high dose DHA + propofol group. Data are presented as mean \pm standard deviation $(\mathrm{n}=10)$. ${ }^{\# \#} \mathrm{P}<0.01$ vs. group $\mathrm{A} ;{ }^{*} \mathrm{P}<0.05,{ }^{* *} \mathrm{P}<0.01$ vs. group $\mathrm{C}$. BDNF, brain-derived neurotrophic factor.

Effects of DHA treatment on acid neurotransmitters. The effects of DHA on the concentration of Glu (Fig. 2A) and GABA (Fig. 2B), and the ratio of Glu/GABA (Fig. 2C) are displayed in Fig. 2. The content of Glu $(P>0.05)$ and GABA $(\mathrm{P}>0.05)$, and the ratio of Glu/GABA $(\mathrm{P}>0.05)$ were not significantly different when comparing group $\mathrm{A}$ with group $\mathrm{B}$. Compared with group A, the content of Glu $(\mathrm{P}<0.01)$ and the ratio of Glu/GABA $(\mathrm{P}<0.01)$ in group $\mathrm{C}$ significantly increased and the GABA content decreased $(\mathrm{P}<0.05)$. Application of DHA in groups $\mathrm{D}, \mathrm{E}(\mathrm{P}<0.05)$ and $\mathrm{F}(\mathrm{P}<0.01)$ led to a decrease in the concentration of Glu and the ratio of Glu/GABA compared with those in group $\mathrm{C}$, and an increase in GABA concentration levels was observed in groups $\mathrm{D}, \mathrm{E}(\mathrm{P}<0.05)$ and $\mathrm{F}(\mathrm{P}<0.05)$ compared with group $\mathrm{C}$. Furthermore, with the increase of dosage of DHA, a dose-dependent effect of DHA was observed. The aforementioned results indicate that DHA may significantly alleviate the learning and memory deficits induced by propofol through balancing the levels of Glu and GABA.

Effects of DHA treatment on SOD and GSH activity, and MDA concentration levels. The effects of DHA treatment on SOD and GSH-Px activity in the hippocampi are displayed in Fig. 3A and B, respectively. SOD and GSH-Px activity were significantly decreased in group $\mathrm{C}$ by a total of $35.1 \%$ $(\mathrm{P}<0.01)$ and $39.8 \%(\mathrm{P}<0.01)$, respectively, compared with control rats. DHA treatment caused a significant increase in SOD and GSH-Px activity in group $\mathrm{E}(\mathrm{P}<0.05)$ and $\mathrm{F}(\mathrm{P}<0.01)$. In addition, with an increase in dose, the effects of DHA on the activity of SOD and GSH-Px were more profound. However, there was no significant difference in SOD or GSH-Px activity between groups $\mathrm{A}$ and $\mathrm{B}$.

The MDA protein concentration levels in the different groups are displayed in Fig. 3C. The results indicated that MDA concentration in propofol-exposed rats was significantly higher compared with those of control rats $(\mathrm{P}<0.01)$. Additionally, treatment with DHA was able to decrease MDA concentration in the hippocampi of rats in groups $\mathrm{D}, \mathrm{E}(\mathrm{P}<0.05)$ 
and $\mathrm{F}(\mathrm{P}<0.01)$ compared with group $\mathrm{C}$. This indicates that treatment with DHA may reduce the oxidative stress caused by propofol-induced anesthesia

Effects of DHA treatment on BDNF. Fig. 4 indicates the effects of DHA treatment on BDNF levels. The BDNF levels of the rats in group $\mathrm{C}$ were decreased by $34.7 \%(\mathrm{P}<0.05)$ compared with those in group A. Treatment with DHA in groups D-F resulted in an increase in the level of BDNF, increasing by $11.3(\mathrm{P}>0.05), 33.9(\mathrm{P}<0.05)$ and 47.8\% $(\mathrm{P}<0.01)$, respectively, compared with group $\mathrm{C}$. There were no statistically significant differences in BDNF levels between groups $\mathrm{A}$ and $\mathrm{B}(\mathrm{P}>0.05)$. The increase in DHA efficacy was dose-dependant based on the aforementioned experimentation.

\section{Discussion}

DHA possesses various potent properties, including neuroprotective and antioxidant effects, in brain tissue, making it an effective molecule for attenuating impairments to learning and memory abilities caused by repeated propofol-induced anesthesia in young rats. The present study revealed that treatment with DHA improved spatial learning and memory through enhancing the activity of antioxidant enzymes, balancing the levels of acid neurotransmitters and increasing the concentration levels of BDNF.

As an established neurological behavior analysis method, MWM testing has become a standard medium by which to assess behavioral neuroscience (24). Therefore, the present experiment selected this technique to explore the effect of DHA treatment on the impairment of learning and memory abilities following repeated propofol-induced anesthesia in young rats. According to the results of MWM testing, after the administration of propofol, the latency of rats in finding the maze platform increased, and the number of platform crossings decreased compared with the control group, suggesting that propofol-induced anesthesia reduces the spatial leaning and memory ability in young rats. The aforementioned data are consistent with previous findings that repeated propofol anesthesia can induce learning and memory impairment via damage to hippocampal neurons $(5,25,26)$. Furthermore, treatment with DHA resulted in an evident decrease in the latency to find the maze platform and a significant increase in the number of platform crossings compared with group $\mathrm{C}$. In addition, the effects of DHA appeared to be dose-dependent. These data suggest that treatment with DHA may effectively improve hippocampus-dependent spatial learning and memory impairment.

To explore the association between learning and memory ability, and amino acid neurotransmitters, the content of Glu and GABA, and the ratio of Glu/GABA were measured in hippocampi tissue samples. Glu is an excitatory amino acid neurotransmitter, whilst GABA is an inhibitory neurotransmitter. Both are key mediators in the central nervous system, and are involved in a variety of brain functions, such as learning and memory, locomotor activity, reproduction and circadian rhythms (27). A balance of neuronal transmission between Glu and GABA is required to maintain normal functions of the brain, including learning and memory (28). The increase of Glu or decrease of GABA (i.e., reduction of the ratio of Glu/GABA) may lead to the impairment of learning and memory (29). In the present study, Glu levels and the Glu/GABA ratio were observed to increase while GABA levels decreased following propofol-induced anesthesia.

Antioxidant systems protect living organisms from oxidative damage through enzymatic or non-enzymatic antioxidant systems and molecules. SOD, GSH-Px and catalase are important enzymes in the aforementioned antioxidant system. An increase of antioxidant enzymes suggests an enhancement of the antioxidant potential of the organs in order to reduce oxidative stress (30). Increased activity of SOD may protect against oxidant impairment (31). In the present study, SOD and GSH-Px activity were significantly increased in the hippocampus following DHA treatment. This may have resulted as a consequent of the body producing more SOD and GSH-Px in the hippocampus in response to oxidative stress induced by propofol exposure. MDA is a product of the lipid peroxidation reaction and is considered to be a parameter for the determination of oxidative damage (32). In the current study, MDA protein concentration levels in the hippocampi of propofol-exposed rats were significantly higher compared with those of control rats, consolidating upon the hypothesis that propofol exposure results in additional oxidative stress. DHA treatment increased SOD activity, however, it also reduced the increase in MDA concentration in propofol-exposed rats. This indicates that DHA is capable of alleviating propofol-induced oxidative impairments.

BDNF is an important molecular mediator of the neuroplasticity of the brain (33) and influences brain functions, such as learning and memory (34). It is known that reductions in BDNF levels in the hippocampus impair learning and memory in animals. In the present study, BDNF levels in the hippocampus were typically lower in the rats with propofol-induced anesthesia compared with group A. Most notably, DHA supplementation caused BDNF levels to increase. Therefore, we hypothesize that the ameliorative effect of DHA on learning and memory ability is associated with increased BDNF levels in the hippocampus.

In conclusion, DHA treatment improved learning and memory impairment by balancing the levels of Glu and GABA, decreasing oxidative damage and increasing BDNF levels in young rats with repeated propofol-induced anesthesia. These results may help the development of a novel therapy for patients suffering from postoperative cognitive dysfunction. Thus, we propose that DHA may be effectively used for postoperative therapy for learning and memory dysfunction induced by repeated propofol anesthesia, however, more studies should be conducted to support this hypothesis.

\section{References}

1. $\mathrm{Hu} \mathrm{ZH}$ and $\mathrm{Ou} \mathrm{YW}$ : Postoperative cognitive dysfunction and Alzheimer's disease from pathogenesis. Med phil 32: 69-71, 2011.

2. Barr G, Anderson RE, Owall A and Jakobsson JG: Being awake intermittently during propofol-induced hypnosis: A study of BIS, explicit and implicit memory. Acta Anaesthesiol Scand 45: 834-838, 2001.

3. Monk TG, Weldon BC, Garvan CW, Dede DE, van der Aa MT, Heilman KM and Gravenstein JS: Predictors of cognitive dysfunction after major noncardiac surgery. Anesthesiology 108: 18-30, 2008. 
4. Wilder RT, Flick RP, Sprung J, Katusic SK, Barbaresi WJ, Mickelson C, Gleich SJ, Schroeder DR, Weaver AL and Warner DO: Early exposure to anesthesia and learning disabilities in a population-based birth cohort. Anesthesiology 110: 796-804, 2009.

5. O'Gorman DA, O'Connell AW, Murphy KJ, Moriarty DC, Shiotani T and Regan CM: Nefiracetam prevents propofol-induced anterograde and retrograde amnesia in the rodent without compromising quality of anesthesia. Anesthesiology 89: 699-706, 1998

6. Erasso DM, Chaparro RE, Quiroga Del Rio CE, Karlnoski R, Camporesi EM and Saporta S: Quantitative assessment of new cell proliferation in the dentate gyrus and learning after isoflurane or propofol anesthesia in young and aged rats. Brain Res 1441: 38-46, 2012.

7. Monk TG, Weldon BC, Garvan CW, Dede DE, van der Aa MT, Heilman KM and Gravenstein JS: Predictors of cognitive dysfunction after major noncardiac surgery. Anesthesiology 108 $18-30,2008$.

8. Zhang H, Wang W, Gao W, Ge Y, Zhang J, Wu S and Xu L: Effect of propofol on the levels of neurotransmitters in normal human brain: A magnetic resonance spectroscopy study. Neurosci Lett 467: 247-251, 2009.

9. Baars JH, von Dincklage F, Reiche J and Rehberg B: Propofol increases presynaptic inhibition of ia afferents in the intact human spinal cord. Anesthesiology 104: 798-804, 2006.

10. Shimizu M, Yamakura T, Tobita T, Okamoto M, Ataka T, Fujihara H, Taga K, Shimoji K and Baba H: Propofol enhances GABA(A) receptor-mediated presynaptic inhibition in human spinal cord. Neuroreport 13: 357-360, 2002.

11. Wang X: Propofol and isoflurane enhancement of tonic gamma-aminobutyric acid type a current in cardiac vagal neurons in the nucleus ambiguous. Anesth Analg 108: 142-148, 2009.

12. Allaouchiche B, Debon R, Goudable J, Chassard D and Duflo F: Oxidative stress status during exposureto propofol, sevoflurane and desflurane. Anesth Analg 93: 981-985, 2001.

13. Kalimeris K, Kouni S, Kostopanagiotou G, Nomikos T, Fragopoulou E, Kakisis J, Vasdekis S, Matsota P and Pandazi A: Cognitive function and oxidative stress after carotid endarterectomy: Comparison of propofol to sevoflurane anesthesia. J Cardiothorac Vasc Anesth 27: 1246-1252,2013.

14. Bazan NG: Neuroprotectin D1 (NPD1): A DHA-derived mediator that protects brain and retina against cell injury-induced oxidative stress. Brain Pathol 15: 159-166, 2005.

15. Martin RE and Bazan NG: Changing fatty acid content of growth cone lipids prior to synaptogenesis. J Neurochem 59: 318-325, 1992.

16. Birch EE, Garfield S, Hoffman DR, Uauy R and Birch DG: A randomized controlled trial of early dietary supply of long-chain polyunsaturated fatty acids and mental development in term infants. Dev Med Child Neurol 42: 174-181, 2000

17. Gamoh S, Hashimoto M, Hossain S and Masumura S: Chronic administration of docosahexaenoic acid improves the performance of radial arm maze task in aged rats. Clin Exp Pharmacol Physiol 28: 266-270, 2001.

18. Fedorova I, Hussein N, Di Martino C, Moriguchi T, Hoshiba J, Majchrzak S and Salem N Jr: An n-3 fatty acid deficient diet affects mouse spatial learning in the Barnes circular maze. Prostaglandins Leukot Essent Fatty Acids 77: 269-277, 2007.
19. Wu A, Ying Z and Gomez-Pinilla F: The salutary effects of DHA dietary supplementation on cognition, neuroplasticity, and membrane homeostasis after brain trauma. J Neurotrauma 28: 2113-2122, 2011

20. Schober ME, Requena DF, Abdullah OM, Casper TC, Beachy J, Malleske D and Pauly JR: Dietary docosahexaenoic acid improves cognitive function, tissue sparing, and magnetic resonance imaging indices of edema and white matter injury in the immature rat after traumatic brain injury. J Neurotraum: 6 Aug, 2015 (Epub ahead of print).

21. Horrocks LA and Farooqui AA: Docosahexaenoic acid in the diet: Its importance in maintenance and restoration of neural membrane function. Prostag Leukot Essent Fatty Acids 70: 4361-4372, 2004

22. Serhan CN, Chiang N and Van Dyke TE: Resolving inflammation: Dual anti-inflammatory and pro-resolution lipid mediators. Nat Rev Immunol 8: 349-361, 2008.

23. Clarke G, O'Mahony S, Malone G and Dinan TG: An isocratic high performance liquid chromatography method for the determination of GABA and glutamate in discrete regions of the rodent brain. J Neurosci Methods 160: 223-230, 2007.

24. D'Hooge R and De Deyn PP: Applications of the Morris water maze in the study of learning and memory. Brain Res Brain Res Rev 36: 60-90, 2001

25. Yu D, Jiang Y, Gao J, Liu B and Chen P: Repeated exposure to propofol potentiates neuroapoptosis and long-term behavioral deficits in neonatal rats. Neuroscience Letters 534: 41-46, 2013.

26. Plaschke K, Schneider J and Kopitz J: Surgery under propofol anesthesia induced behavioral changes associated with increased cerebral apoptosis in rats. J Liver 2: 136-145, 2013.

27. Jinno S and Kosaka T: Neuronal circuit-dependent alterations in expression of two isoforms of glutamic acid decarboxylase in the hippocampus following electroconvulsive shock: A stereology-based study. Hippocampus 19: 1130-1141, 2009.

28. Car H and Wiśniewski K: Similarities and interactions between GABAergic and glutaminergic systems. Rocz Akad Med Bialymst 43: 5-26, 1998.

29. Matsuyama S, Taniguchi T, Kadoyama K and Matsumoto A: Long-term potentiation-like facilitation through GABAA receptor blockade in the mouse dentate gyrus in vivo. Neuroreport 19: 1809-1813, 2008.

30. She JQ, Wang M, Zhu DM, Tang M, Chen JT, Wang L and Ruan DY: Monosialoanglioside (GM1) prevents lead-induced neurotoxicity on long-term potentiation, SOD activity, MDA levels, and intracellular calcium levels of hippocampus in rats. Naunyn Schmiedebergs Arch Pharmacol 379: 517-524, 2009.

31. Tang XL, Qiu Y, Turrens JF, Sun JZ and Bolli R: Late preconditioning against stunning is not mediated by increased antioxidant defenses in conscious pigs. Am J Physiol 273: H1651-H1657, 1997.

32. Pompella A: Biochemistry and histochemistry of oxidant stress and lipid peroxidation. Int J Vitam Nutr Res 67: 289-297,1997.

33. McAllister AK, Katz LC and Lo DC: Neurotrophins and synaptic plasticity. Annu Rev Neurosci 22: 295-318, 1999.

34. Tyler WJ, Alonso M, Bramham CR and Pozzo-Miller LD: From acquisition toconsolidation: on the role of brain-derived neurotrophic factor signaling in hippocampal-dependent learning. Learn Mem 9: 224-237, 2002. 